\title{
Optimization of Recloser Placement in DG-Enhanced Distribution Networks Using a Multi-objective Optimization Approach
}

\author{
Fabian Lopez ${ }^{1}$ and Andrés Pantoja ${ }^{1}$ \\ ${ }^{1}$ Department of Electronics \\ Universidad de Nariño \\ Ciudadela Universitaria Torobajo, Pasto, Colombia \\ e-mail: fabianlopezch@outlook.com, ad_pantoja@udenar.edu.co
}

\begin{abstract}
Efficient placement of protective devices in electric power distribution networks is necessary in order to achieve a reliable system and provide continuous power supply to customers as long as possible. The islanded operation with distributed generation (DG) provides a way to reduce the energy not supplied (ENS) but the placement of protections, such as reclosers, is necessary in order to allow the system to achieve this mode of operation. This paper presents a multi-objective optimization method to place efficiently normally closed reclosers by using a constrained non-dominated sorting genetic algorithm (C-NSGA-II) to reduce SAIDI, ENS and investment costs. A co-simulation approach is used in such a way that the power system is modelled in PowerFactory, while MATLAB is used to implement the CNSGA-II. Then, a distribution test network is probed in simulation cases with different DG penetration levels, showing the efficiency of the proposed optimization method. Results show the importance of protective devices and DG in enhancing system reliability and reducing the energy not supplied to customers.
\end{abstract}

\section{Key words}

Co-simulation, distributed generation, distribution systems reliability, multi-objective optimization, protective systems.

\section{Nomenclature}

DG: Distributed Generation.

ENS: Energy Not Supplied.

SAIDI: System Average Interruption Duration Index

NCR: Normally Closed Recloser

SOOP: Single Objective Optimization Problem

MOOP: Multi-Objective Optimization Problem

NCD: Non-Constraint Dominated

$A C I T_{i}$ : average customer interruption time of the load point $i$.

$P d_{i}$ : amount of power not supplied when load point $i$ is disconnected.

$k$ : total number of loads in the network.

$E U A C$ : Equivalent Uniform Annual Cost.

$A O C$ : Annual Operation Cost.

$R_{p}$ : Cost of a single recloser.

$N_{r}$ : Amount of placed reclosers.

$d_{r}$ : Discount rate.

$l_{t}$ : Lifetime of reclosers

\section{Introduction}

A priority of any distribution utility is to meet the requirements for safety, reliability and quality of supply. This can be achieved with the assist of some subsystems associated with the distribution network, where the protection system is the most important due to its capability for clearing and isolating faults. Concerning reliability, satisfying the load demand of customers with a continuous supply of energy is required to improve the reliability indices and reduce the energy not supplied (ENS). To make it possible, distribution automation is necessary to isolate faults on the network as quickly as possible, offering the possibility that distributed generation (DG) units can operate in islanded mode, an important function required for future smart grids [1].

In order to achieve a proper balance between financial resources and the technical requirements of the network, a cost/benefit analysis of the protection system must be considered. With these conditions, distribution utility has the task to meet two conflictive objectives: minimizing economical cost and maximizing reliability. Fulfilling the above objectives, the number and location of protective devices are critical variables. Thus, the contribution of this work is to deal with the planning of protection systems based on normally closed reclosers (NCRs). These devices are capable to isolate faulted sections and can be used to allow the islanded operation of networks with DG by establishing autonomous shares and achieving service restoration to certain loads of the distribution network.

The optimal placement of protective devices has been studied as single objective (SOOP) and multi-objective (MOOP) optimization problems taking into account the DG capability of islanded operation. Considering the SOOP approach, the reactive tabu search algorithm (RTS) is implemented in [2] to solve a cost minimization problem modeled through a mixed integer non-linear programming with real and binary variables. Moreover, [3] presents a novel structure for the simple differential evolution algorithm (DE) to solve optimal recloser placement by minimizing an objective function composed by four 
reliability indices. Taking into account both optimal positions for DG and protection devices, in [4] the feeder is equipped with capacity-constrained DG, while a custom-tailored genetic algorithm is used to minimize a composite reliability index.

The aforementioned studies address the reliability optimization problems with one of two approaches: $i$ ) minimize cost while satisfying reliability constraints, and ii) minimize customer interruptions subject to cost constraints [5]. Nevertheless, decision-making in planning of protection systems should be treated as a multi-objective optimization problem because of the conflictive maximization of reliability and minimization of costs. To work with the MOOP approach, two ways can be considered. On the one hand, the MOOP is turned into a SOOP by means of methods such as the weighted-sum approach and the $\varepsilon$ constraint method [6]. However, such a transformation is often subjective to the decision-maker and it needs precise information about the trade-off relationship among objectives. On the other hand, a more reliable approach is to first find a Pareto-optimal set with multiple trade-off solutions, and then, chose one solution from the set using convenient information or assumptions about the system.

Considering this approach, there are some works using the Pareto-optimal concept in planning of protection systems. A methodology for multi-objective optimization of protective devices allocation in order to minimize, SAIDI, SAIFI and $\mathrm{MAIFI}_{\mathrm{E}}$ indices simultaneously is proposed in [7]. The authors use the non-dominated sorting genetic algorithm (NSGA-II) in the search of the best solutions, in the same way as in [8], where the problem address with the minimization of the installation cost of sectionalizers while improving SAIDI and ECOST indices. With a different metaheuristic, the multi-objective ant colony system (MACS) is used in [9] for optimal placement of switching and protective devices in distribution systems by means of minimizing SAIFI, SAIDI, and total cost.

In this paper, we propose a multi-objective optimization that uses the Pareto-dominance concept to minimize SAIDI, ENS, and cost investment simultaneously on a DGenhanced distribution network with islanded operation capabilities. The optimization procedure provides multiple trade-off solutions of efficient configurations with different amounts and placements of NCRs in the distribution network. Since the optimal placement of protective devices is a complex combinatorial problem with nonlinearity, discontinuity, and non-differentiability characteristics [4], [9], [10], solving the problem with traditional methods (e.g., linear and nonlinear programming) is difficult. Then, the use of metaheuristic methods such as the Non-dominated Sorting Genetic Algorithm (NSGA-II) [11] is an appropriate alternative to attain efficient solutions. To obtain a solution set belonging to a Pareto surface, we use a linked simulation with MATLAB and PowerFactory with the automatic data exchange procedure presented in [12].

\section{General Optimization Problem}

\section{A. Multi-objective Optimization and Pareto Optimality}

Even though many of the decision-making problems are handled as single objective problems, including all the aspects in a single function is difficult. Thus, a multiobjective optimization problem can be defined with a vector of functions that includes a number of conflicting objectives to be maximized or minimized. Without loss of generality it is assumed that these objectives are to be minimized, as such the problem can be expressed by its general form as follow [6]:

$$
\begin{aligned}
& \text { Minimize } \quad f_{m}(X), \quad m=1,2, \ldots, M \text {; } \\
& \text { subject to } \quad g_{j}(X) \geq 0, \quad j=1,2, \ldots, J \text {; } \\
& h_{k}(X)=0, \quad k=1,2, \ldots, K \text {; } \\
& x_{i}^{(L)} \leq x_{i} \leq x_{i}^{(U)}, \quad i=1,2, \ldots, n .
\end{aligned}
$$

where $M, J$ and $K$ are the number of objective functions, inequality, and equality constraints, respectively, and $X=\left(x_{1}, x_{2}, \ldots, x_{n}\right)^{T}$ is a vector of the $n$ decision variables. The values $x_{i}^{(L)}$ and $x_{i}^{(U)}$ define the lower and upper bounds of the decision space that restricts the decision variables.

In MOOPs where the objectives are in conflict to each other, there does not exists a single solution that simultaneously optimizes all objective functions. Instead, the most commonly adopted concept for optimality in MOOPs is the Pareto optimality [13], where a feasible vector of decision variables $X^{*}$ is Pareto optimal if there does not exist another $X$ such that $f_{i}(X) \leq f_{i}\left(X^{*}\right)$ for all $i=1,2, \ldots, M$ and $f_{j}(X)<f_{j}\left(X^{*}\right)$ for at least one $j$. Almost always, this approach gives a set of multiple trade-off solutions instead of a single solution. That set of solution is so-called the Pareto-optimal set and the vectors $X^{*}$ belonging to this set are so-called non-dominated solutions. The image of the Pareto-optimal set under the objective functions is the Pareto front.

Since multiple solutions arise in MOOPs, a mechanism to assess their relative fitness must be established. Thus, the Pareto-dominance concept has widely been used to accomplish this task and guide the search towards the Pareto front. The conditions that must be true for a solution $X^{(1)}$ to dominate other solution $X^{(2)}$ are defined as follow [6]:

1. The solution $X^{(1)}$ is no worse than $X^{(2)}$ in all objectives.

2. The solution $X^{(1)}$ is strictly better than $X^{(2)}$ in at least one objective.

Since the Pareto-dominance is a method to compare solutions in MOOPs, this concept is used in most multiobjective optimization algorithms to search for nondominated solutions and achieve the Pareto front.

\section{B. Islanded Mode of DG}

The islanded operation capability is one of the most outstanding features of a DG-enhanced distribution network because it may offer a reliable and uninterrupted energy supply to certain loads. This feature allows the system to produce microgrids with the ability to operate in off-grid mode when faults arise in the utility grid. It can be 
enabled by using switches and protective devices (e.g. NCRs). Although islanded mode is not widely accepted, implementation of intentional islanding of DG is a current research area covered by standards such as the IEEE 15472003, continuously under consideration for future revisions [14].

Considering disturbances, when a fault arises in a DGenhanced distribution network, it may be energized from both the substation transformer and the DG whereby miscoordination between protective devices may emerge. This condition increases the difficulty in the reliability assessment. In this case, in normal conditions we assume that the distribution network works as a typical radial network, and that the DG only supplies power when a fault occurs in the feeder. This assumption is established given that the focus of this work is to analyze the improvement that DG can provide in system reliability. In consequence, for a fault anywhere on the network, only the closest recloser upstream to the fault can operate.

With the aforementioned assumptions, the DG-enhanced radial feeder shown in Figure 1 is used as an example to illustrate the islanded operation mode. In normal conditions, the utility grid is the only source that supplies energy to loads $L_{1}, L_{2}$ and $L_{3}$, while the DG does not provide power. Now, assume that a fault arise between busbars $B_{1}$ and $B_{2}$ which causes the opening of the recloser $(\mathbf{R})$. After this, loads $L_{2}$ and $L_{3}$ are isolated from the utility grid forming an island along with the DG unit. DG should be able to provide energy to total load $L_{T}=L_{2}+L_{3}$, while the network returns to normal conditions. When the island is established, one of the following cases may occur: $i$ ) the DG power supply $\left(P_{D G}\right)$ equals $L_{T}$ power consumption $\left(P_{T}\right)$, which need no actions and is the most efficient case, ii) $P_{D G}$ is not sufficient for meet $P_{T}\left(P_{D G}<P_{T}\right)$, so load shedding is required in order to keep the supply-demand balance and causing an increase in ENS, and iii) $P_{D G}$ exceeds $P_{T}\left(P_{D G}>P_{T}\right)$, so actions such as storing/dumping the excess of energy, or reducing generation should be considered.

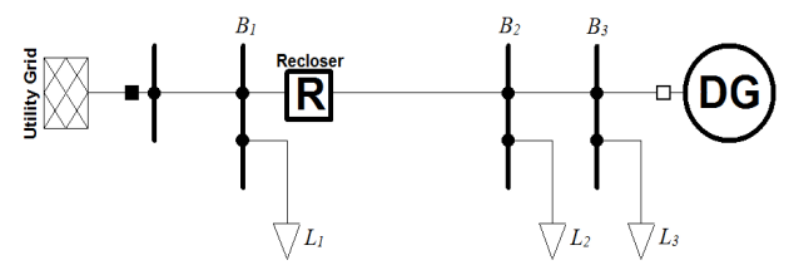

Figure 1. Illustrative DG-enhanced feeder.

Taking into account the above, an inadequate placement of reclosers may result in the formation of inefficient islands either with excessive or insufficient generation, so the reliability provided by DG is not obtained. Hence, efficient placement of reclosers is required in order to ensure efficient islands, and in consequence, maximizing the benefits of using DG.

\section{Problem Formulation}

One of the purposes of the protective devices is to enhance certain reliability indices, procuring a continuous energy supply to loads. To accomplish this goal, investments must be done depending on the desired performance of the distribution utility. This work addresses SAIDI and ENS reduction in the optimization problem, along with a cost function that includes investment and operational costs of the protective system. SAIDI is selected since in addition to being the most common reliability index used by utilities, reliability investments based on this index alone have shown good improvements in SAIFI and $\mathrm{MAIFI}_{\mathrm{E}}$ too [5]. Also, since indices such as SAIFI depend on the interruptions that customers perceive, poor improvements could be achieved with the approach used in this research because interruptions can be experienced by customers while the islands are established.

This problem has conflictive functions because a high improvement in reliability usually requires high investment, and inexpensive protection systems may lead to poor reliability indices. Therefore, there does not exist a single solution that simultaneously minimizes the three objective functions so a Pareto optimization is used to find a set of trade-off solutions. The MOOP is defined based on the general form described in (1):

$$
\begin{array}{cc}
\min & \operatorname{SAIDI}(X), \operatorname{ENS}(X), \operatorname{EUAC}(\mathrm{X}) \\
\text { subject to } & 1-\frac{\operatorname{EUAC}(\mathrm{X})}{\operatorname{EUAC} \text { max }} \geq 0, \\
x_{i} \in\{0,1\}
\end{array}
$$

where,

$$
\begin{aligned}
S A I D I & =\frac{\sum_{i=1}^{k} A C I T_{i} C_{i}}{\sum_{i=1}^{k} C_{i}} \\
E N S & =\sum_{i=1}^{k} A_{C I T_{i} P d_{i}} \\
E U A C & =A O C+\left[\frac{d_{r}\left(1+d_{r}\right)^{l_{t}}}{\left(1+d_{r}\right)^{l_{t}}-1}\right] R_{p} N_{r}
\end{aligned}
$$

Here, $A C I T_{i}$ is the average customer interruption time of the load point $i, C_{i}$ is the number of customers in load point $i, P d_{i}$ is the amount of power not supplied when load point $i$ is disconnected, and $k$ is the total number of loads in the network. Considering costs, EUAC is the equivalent uniform annual cost, where $A O C$ is the annual operation cost, $R_{p}$ is the cost of a single recloser, $N_{r}$ is the amount of placed reclosers on the network and the expression in the brackets represents an annualization factor composed by a discount rate $d_{r}$ and the lifetime of reclosers $l_{t}$. Also, $E U A C_{\max }$ is the maximum $E U A C$ that the utility is willing to invest in reliability improvement. By last, the $n$ decision variables composing the vector $X=\left(x_{1}, x_{2}, \ldots, x_{n}\right)^{T}$ are binary variables which represent the existence $\left(x_{i}=1\right)$ or absence $\left(x_{i}=0\right)$ of a recloser in the $i^{t h}$ branch of the network.

\section{Constrained Non-Dominated Sorting Genetic Algorithm-II (C-NSGA-II)}

Problems related to optimal placement of protection devices have nonlinear, discontinuous, and nondifferentiable characteristics so the use of traditional methods may be infeasible. Furthermore, the outcome of 
classical optimization methods is a single solution and not a set of solutions, as required in multi-objective problems. Thus, a feasible alternative to overcome this issue is to use a multi-objective evolutionary algorithm (MOEA). A MOEA use a population based approach along with the Paretodominance concept to look for multiple non-dominated solutions. In this paper, we use the constrained nondominated sorting genetic algorithm (C-NSGA-II) [11] in order to search for solutions of the MOOP defined in (2). The C-NSGA-II use the same two key features of NSGA-II: i) a fast non-dominated sorting approach to sort each solution within a non-dominated front $\mathcal{F}_{d}(d=1,2, \ldots)$ based on an assigned rank $d$, and ii) a crowding distance metric used to preserve diversity among solutions. The main difference is that in C-NSGA-II the usual definition of domination is replaced by a constrain-domination condition. In this sense, to say that a solution $X^{(1)}$ constrain-dominate a solution $X^{(2)}$, any of the following conditions must be true:

1. Solution $X^{(1)}$ is feasible and solution $X^{(2)}$ is not.

2. Solution $X^{(1)}$ and $X^{(2)}$ are both infeasible, but solution $X^{(1)}$ has smaller constraint violation.

3. Solution $X^{(1)}$ and $X^{(2)}$ are feasible, and $X^{(1)}$ dominates the solution $X^{(2)}$ in the usual domination sense defined in Section 3A.

To illustrate these concepts, a pseudo-code of the proposed C-NSGA-II is shown in Algorithm 1. First, a random initial population $P_{0}$ is created composed by individuals of $N$ chromosomes as in $X_{N}$ (i.e., the decision vectors). Each binary string $X_{N}$ is used to place reclosers in the distribution network based on the binary value of each decision variable $\left(x_{n}\right)$. Next, a reliability assessment is done in PowerFactory for each configuration to obtain the objective function values. Then, the solutions in the population are sorted into $d$ non-constrain-dominated (NCD) sets $\left(\mathcal{F}_{d}\right)$ using the constrain-domination condition, such that, the front $\mathcal{F}_{i}$ is better that $\mathcal{F}_{j}$ if $i<j$. Also, crowding distance is calculated for solutions in each front $\mathcal{F}_{d}$. After this, a selection process is applied to the population by means of a constrained tournament. With this selection operator, given two solutions $X^{(1)}$ and $X^{(2)}$, solution $X^{(1)}$ is chosen if any of the following conditions are true:

1. Solution $X^{(1)}$ belongs to a better NCD set.

2. Solutions $X^{(1)}$ and $X^{(2)}$ belong to the same NCD set, but solution $X^{(1)}$ has greater crowding distance value.

The offspring population $Q_{0}$ is produced by regular genetic operators such as crossover and mutation. When the offspring population is obtained, a new population $R_{t}=$ $P_{t} \cup Q_{t}$ is created. The objective functions evaluation with solutions in $R_{t}$ is accomplish through reliability assessment again. Then, $R_{t}$ is sorted in NCD fronts in order to obtain the population of the next generation. Thus, when $R_{t}$ is sorted, the population $P_{t+1}$ is filled by the elements inside of front $\mathcal{F}_{1}$ (the best front) only if the addition of the size of $P_{t+1}$ and the size of $\mathcal{F}_{1}$ is less than $N$. The aforementioned process is repeated with subsequent fronts until the value of $P_{t+1}$ size added to $\mathcal{F}_{j}$ size is greater than $N$. When it happens, $P_{t+1}$ cannot include all solutions contained in $\mathcal{F}_{j}$. Hence, only the $N-\left|P_{t+1}\right|$ solutions with the greatest crowding distance values from $\mathcal{F}_{j}$ are selected to complete the population of $P_{t+1}$. Finally, offspring population $Q_{t+1}$ is created by applying constrained tournament selection, crossover and mutation operators to $P_{t+1}$ population.

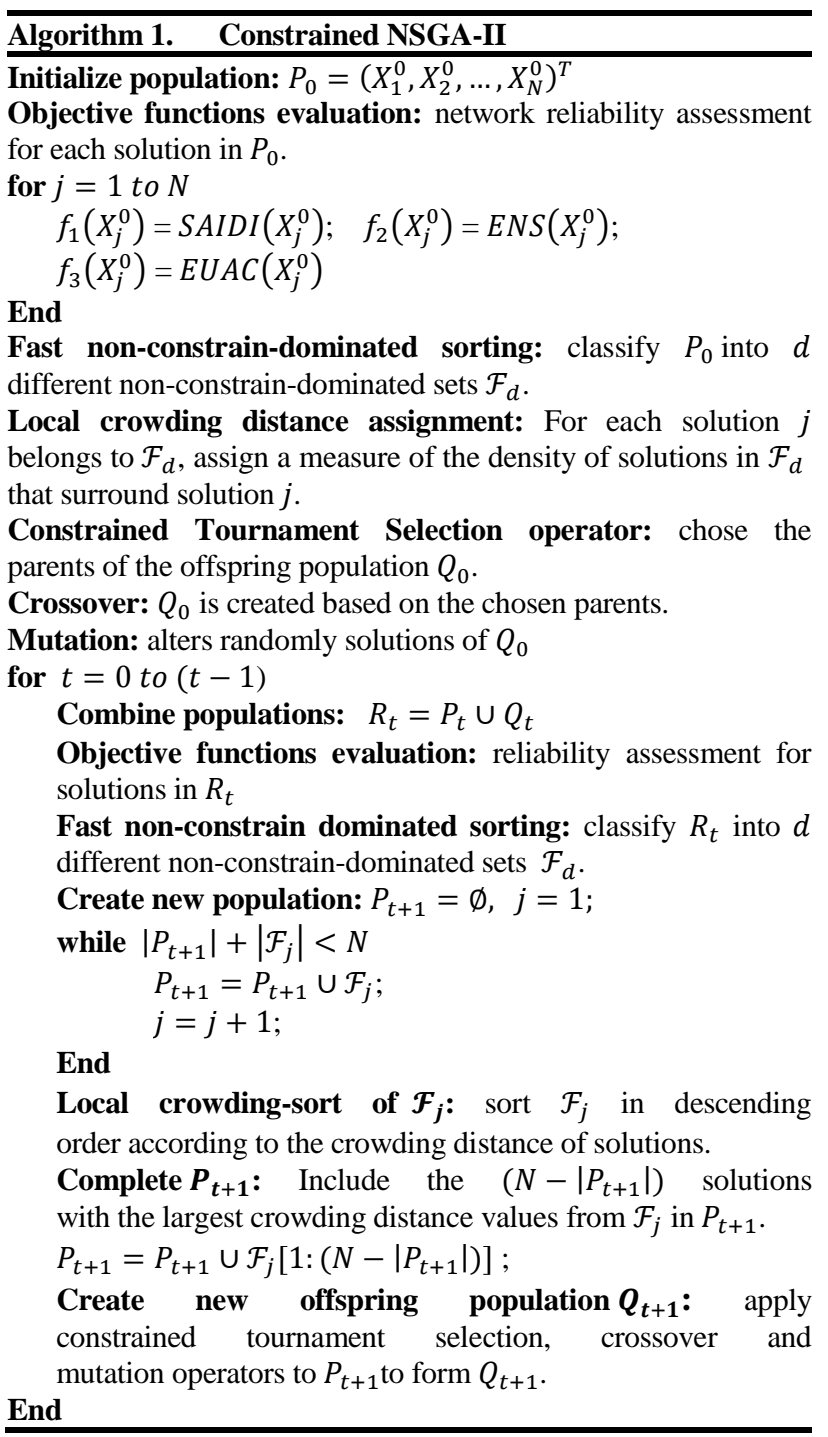

The use of powerful tools offered by PowerFactory in the evaluation of the objective functions is a remarkable aspect of this work. Although optimization can be done in the same software, the programming platform of PowerFactory presents some difficulties and coding the optimization algorithm could become a hard task. In this sense, the co-simulation approach used in this work takes advantage of an easy programming environment such as MATLAB and its toolboxes to develop the optimization algorithm.

\section{Case Study and Results}

\section{A. Case Study}

To assess the performance of the proposed optimization algorithm and show its application, we use the network at bus 6 of the Roy Billinton Test System (RBTS) implemented in PowerFactory (Figure 2) based on the 
system parameters presented in [15]. This network has 41 load points with a peak load of $20 \mathrm{MW}$ and two voltage levels $(33 \mathrm{kV}$ and $11 \mathrm{kV})$. The original radial network has been enhanced with DG penetration in order to make comparative simulations between the original network and the enhanced one with DG penetration of $20 \%$ and $60 \%$.

A failure model is required to accomplish the reliability analysis, so we define a stochastic failure model for the network components, using the data detailed in [16]. Here, the failure rates and repair times for transformers, breakers, busbars, and lines are listed for each voltage level. Regarding $E U A C$, the values of $A O C, R p, d r$ and $l t$ are set to 400 USD, 18000 USD, 0.1 (10\%), and 20 years, respectively, so the $E U A C$ for a single installed recloser is 2514 USD, and EUACmax is set to 26000 USD.

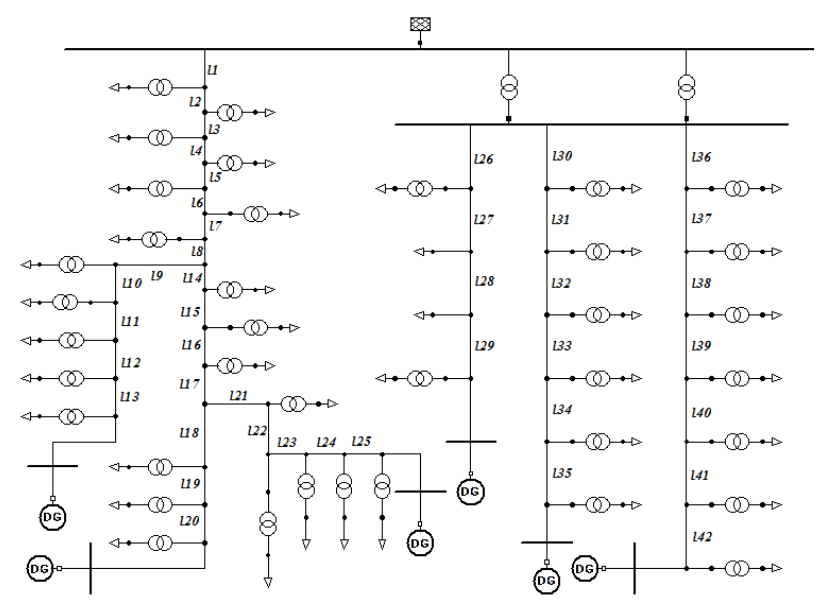

Figure. 2. Distribution Network at Bus 6 of the RBTS with DG Penetration.

\section{B. Results}

The C-NSGA-II has been implemented in MATLAB for finding efficient placement of reclosers in the distribution network described above (Figure 2). The network is simulated in PowerFactory to calculate the objective functions (SAIDI and ENS) according to the stochastic model of failures and the power flows. The optimization procedure results in the set of solutions showed in Figure 3, which represent three simulation cases, one without DG penetration and two with different levels of DG penetration.

For each simulation case, we have selected three representative non-constrain-dominated solutions presented in Table I. Between these solutions, "A" represents the cheapest solution (EUAC $=\$ 7542$ ), but it produces less improvements in SAIDI and ENS. On the other hand, solution " $\mathrm{C}$ " has the best SAIDI and ENS values, but it requires the highest investment (EUAC $=\$ 25140$ ), satisfying the constraint of the problem. By last, "B" represents an affordable solution (EUAC $=\$ 15084$ ) with intermediate SAIDI and ENS values with respect to solutions " $A$ " and "C".

Observing Figure 3, it is evident that SAIDI and ENS are non-linear functions of the number of recloser placed in the network. Even more, the extent of improvement in those indices, achieved by the addition of reclosers, decrease as the amount of reclosers increases. From this point of view, having multiple solutions is useful to analyze when increases in investment does not produce significant decrease in SAIDI and ENS values. Then, it is possible to find points where the benefits obtained by the addition of a recloser do not justify the investment.

Another important result with the addition of DG and isolated mode capability is that solutions are displaced towards lower values of SAIDI and ENS as the DG penetration increases.

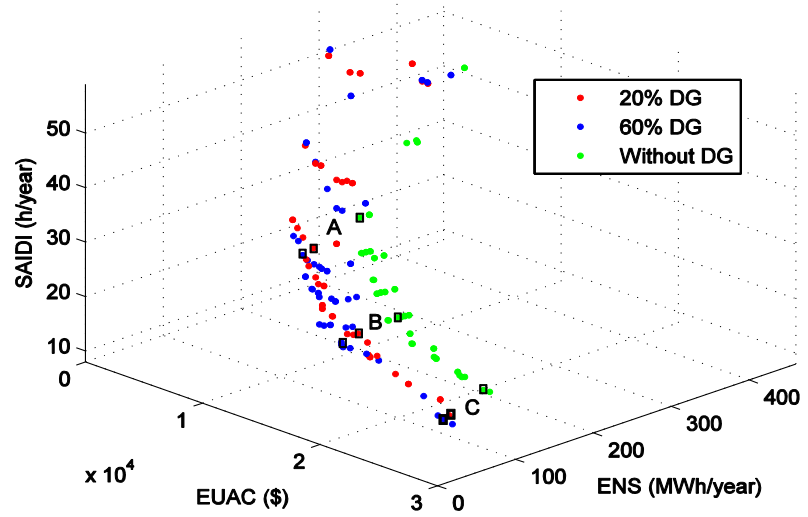

Figure. 3. Sets of Non-Constrain-Dominated Solutions

Analyzing the positions in which reclosers are placed (Table I), it may be noted that feeders are segmented efficiently depending on the number of reclosers to be installed in the network.

Regarding DG penetration, the optimization process seeks for solutions locating reclosers close to DG units with the aim to create microgrids. As an example, consider the solution " $C$ " in which the recloser placed in the linesegment $l 17$ for case $i$. is relocated in line-segments $l 22$ and $l 21$ for cases ii. and iii, respectively. This allows the formation of an isolated zone (microgrid), with few loads downstream the recloser. When permanent faults arise upstream, a DG unit can supply energy inside this isolated zone, and thus, the system reliability is improved.

Table I. - Samples of non-dominated solutions of C-NSGA-II

\begin{tabular}{cccccc}
\hline \hline CASE & $\begin{array}{c}\text { SAIDI } \\
(\mathbf{h} / \mathbf{a})\end{array}$ & $\begin{array}{c}\text { ENS } \\
(\mathbf{M W} / \mathbf{h})\end{array}$ & $\begin{array}{c}\text { EUAC } \\
\mathbf{( \$ )}\end{array}$ & Reclosers Position \\
\hline \hline & $\mathrm{A}$ & 28.65 & 238.35 & 7542 & $l 2, l 30, l 36$ \\
$i .0 \%$ & $\mathrm{~B}$ & 19.15 & 173.23 & 15084 & $l 2, l 9, l 14, l 26, l 30, l 37$ \\
DG & $\mathrm{C}$ & 15.41 & 132.13 & 25140 & $l 2, l 9, l 14, l 17, l 26$, \\
& & & & $l 29, l 30, l 32, l 36, l 40$ \\
\hline & $\mathrm{A}$ & 25.94 & 178.78 & 7542 & $l 7, l 30, l 37$ \\
ii. 20\% & $\mathrm{B}$ & 18.70 & 122.57 & 15084 & $l 3, l 15, l 26, l 31, l 39, l 42$ \\
$\mathrm{DG}$ & $\mathrm{C}$ & 12.97 & 88.16 & 25140 & $l 3, l 9, l 15, l 22, l 26$, \\
& & & & $l 30, l 32, l 36, l 39, l 42$ \\
\hline & $\mathrm{A}$ & 25.25 & 165.38 & 7542 & $l 8, l 30, l 36$ \\
iii. 60\% & $\mathrm{B}$ & 17.70 & 101.44 & 15084 & $l 7, l 16, l 27, l 31, l 36, l 40$ \\
$\mathrm{DG}$ & $\mathrm{C}$ & 12.57 & 81.04 & 25140 & $l 7, l 9, l 21, l 27, l 31$, \\
& & & & $l 32, l 35, l 36, l 39, l 41$ \\
\hline \hline
\end{tabular}

Finally, by comparing solutions of the cases ii. and iii. having the same investment (i.e. similar EUAC), evidently case iii has solutions with better SAIDI and ENS values than those of case ii. As an example, comparing solution 
"B" of case ii (SAIDI=18.70, ENS=122.57) with "B" of case iii (SAIDI=17.70, ENS=101.44), clearly the values for case iii are lower than those in case ii. Therefore, the solution of case iii is better than the one of case ii. In consequence, the aforementioned results show that best solutions can be found as the level of DG increases.

\section{Conclusion}

A methodology that uses a multi-objective approach has been used to address the problem of protection planning of distribution systems. The aim of this method is to find a set of multiple trade-off solution that determine efficient number and placement of reclosers in a distribution network. Those solutions are searched in a way that SAIFI, ENS and EUAC are minimized based on the Pareto dominance concept.

The C-NSGA-II is implemented and applied in the optimization process to find a set of non-constraindominated solutions satisfying the constraint established in the optimization problem.

The importance of the protective devices and DG placement has been proved in enhancing reliability and reducing the energy not supplied by means of the results in the simulation cases. Moreover, the optimization of reclosers placement procure the creation of microgrids when islanded mode of DG is allowed. Besides, feeders are efficiently segmented based on the number of reclosers to be placed, and lower SAIDI and ENS values are achieved as the DG penetration level increases. Thus, better non-constrain-dominated solutions can be found.

Coupling of two powerful tools, such as MATLAB and PowerFactory, shows a good approach to solve complex optimization problems related with power systems, as the proposed in this work.

Future work includes developing a methodology to prove by simulation that the solutions found in the C-NSGA-II algorithm belong to the Pareto front and that there are no better solutions in the neighborhood of this front.

\section{Acknowledgement}

We would like to thank ALTERNAR Project, BPIN 20130001000089, Acuerdo 005 de 2013, OCAD - Fondo de CTel - SGR, Colombia and 'Programa de Jovenes Investigadores e Innovadores - 2016' of Gobernación de Nariño and CEIBA foundation, for the partial support of this work.

\section{References}

[1] M. Brenna, F. Foiadelli, P. Petroni, G. Sapienza, and D. Zaninelli. Distributed generation regulation for intentional islanding in smart grids. In 2012 IEEE PES Innovative Smart Grid Technologies (ISGT), pp 1-6, 2012.

[2] L. G. W. da Silva, R. A. F. Pereira, J. R. Abbad, and J. R. S. Mantovani. Optimised placement of control and protective devices in electric distribution systems through reactive tabu search algorithm. Electric Power Systems Research, Vol. 78,
No.3, pp.372-381, 2008.

[3] M. Hajivand, R. Karimi, M. Karimi, et al. Optimal recloser placement by binary differential evolutionary algorithm to improve reliability of distribution system. International Journal of Information, Security and SystemsManagement, Vol. 3, No. 2, pp.345-349, 2014.

[4] A. Pregelj, M. Begovic, and A. Rohatgi. Recloser allocation for improved reliability of dg-enhanced distribution networks. IEEE Transactions on Power Systems, Vol. 21, No. 3, pp.14421449, 2006.

[5] R. E. Brown. Electric power distribution reliability. CRC press, 2008.

[6] K. Deb. Multi-objective optimization using evolutionary algorithms, volume 16. John Wiley \& Sons, 2001.

[7] G. D. Ferreira, A. S. Bretas, and G. Cardoso. Optimal distribution protection design considering momentary and sustained reliability indices. In Modern Electric Power Systems (MEPS), 2010 Proceedings of the International Symposium, pages 1-8. IEEE, 2010

[8] M. R. Mazidi, M. Aghazadeh, Y. A. Teshnizi, and E. Mohagheghi. Optimal placement of switching devices in distribution networks using multi-objective genetic algorithm nsgaii. In 2013 21st Iranian Conference on Electrical Engineering (ICEE), pages 1-6. IEEE, 2013.

[9] W. Tippachon and D. Rerkpreedapong. Multiobjective optimal placement of switches and protective devices in electric power distribution systems using ant colony optimization. Electric Power Systems Research, Vol. 79, No. 7, pp.1171-1178, 2009.

[10] J.-H. Teng and Y.-H. Liu. A novel acs-based optimum switch relocation method. IEEE transactions on power systems, Vol. 18, No. 1, pp.113-120, 2003.

[11] K. Deb, A. Pratap, S. Agarwal, and T. Meyarivan. A fast and elitist multiobjective genetic algorithm: Nsga-ii. IEEE transactions on evolutionary computation, Vol. 6, No. 2 , pp.182-197, 2002.

[12] A. Stativ a, M. Gavrilas, and V. Stahie. Optimal tuning and placement of power system stabilizer using particle swarm optimization algorithm. In Electrical and Power Engineering (EPE), 2012 International Conference and Exposition on, pages 242-247. IEEE, 2012.

[13] C. A. C. Coello. Recent trends in evolutionary multiobjective optimization. In Evolutionary Multiobjective Optimization, pages 7-32. Springer, 2005.

[14] IEEE Std. 1547-2003, IEEE Standard for interconnecting Distributed resources with electric power systems, 2003.

[15] R. Billinton and S. Jonnavithula. A test system for teaching overall power system reliability assessment. IEEE Transactions on Power Systems, Vol. 11, No. 4, pp.1670-1676, 1996.

[16] R. N. Allan, R. Billinton, I. Sjarief, L. Goel, and K. So. A reliability test system for educational purposes-basic distribution system data and results. IEEE Transactions on Power Systems, Vol. 6, No. 2, pp.813-820, 1991. 